\title{
3D Dynamic Visualization Simulation System of Pool Fire Triggered by Major Hazard Installation
}

\author{
Wenjiang Chen ${ }^{1,2}{ }^{*}$, Zhaoji $\mathrm{Hu}^{1}$, Yan Yong ${ }^{2}$, Hongbo $\mathrm{Su}^{2}$ \\ ${ }^{1}$ School of Resources, Environmental \& Chemical Engineering, Nanchang University, Nanchang 330031, \\ China. \\ 2 Department of Civil, Environmental \& Geomatics Engineering, Florida Atlantic University, FL 33431, USA. \\ * Corresponding author. Tel.: +86 15970658121; +1 9549521784; email: chenwenjiang@ncu.edu.cn; \\ chenw@fau.edu. \\ Manuscript submitted January 10, 2018; accepted March 8, 2018. \\ doi: 10.17706/jsw.13.9.481-496
}

\begin{abstract}
This paper proposes a method of three-dimensional (3D) dynamic visualization simulation of pool fire triggered by major hazard installation. The method describes the 3D dynamic characteristics of pool fire by establishing the 3D model which combines with the real-time data of various parameters including environment and position information of people. To improve the computational efficiency of the model and enhance the sensing cognition of accidents, a 3D simulation system of pool fire is developed by 3D visualization technology. And an application of the system is performed to simulate the real-time process and consequences of pool fire. The application results show that the 3D simulation method of pool fire is of real-time, lifelike and visual characteristics, which not only embodies the great practical application value of 3D visualization technology in the field of safety engineering, but also provides references for safety management, disaster prediction, risk assessment, emergency response and safety planning of petrochemical enterprises.
\end{abstract}

Key words: Particle system, pool fire, virtual reality, visualization.

\section{Introduction}

With the rapid development of the economy, many large chemical industrial parks have been built in rapid succession. There are many major hazard installations (MHI) storing large amount of flammable \& explosive substances in a chemical industrial park. With improper management of them, MHIs are likely to cause major accidents, such as fire and explosion, which have a strong impact on the surrounding area.

To improve the safety management of MHIs and the capacity of accident emergency rescues, some methods have been developed to predict the consequences of accidents [1]. Adedigba et al. [2] presented a new non-sequential barrier-based process accident model to predict the probability of an accident. Khalil [3] developed a probabilistic model to quantify occupational risks of fire and explosion events initiated by leaks that ignite within enclosed spaces. Bubbico and Mazzarotta [4] presented a simplified mathematical model to assess the consequences during the evolution of the accident. These models and methods focused on predicting the probability of an accident or consequence assessment according to the key parameters in a specific condition. The semi-empirical model was also introduced into consequence assessment in petrochemical industry [4], but the assessment results of consequences were static under the constant state parameters. However, the consequences of accidents dynamically vary with the environmental parameters 
and the characteristic parameters of MHIs. These parameters will also affect the occurrence and evolution of accidents. In other words, the assessment results are real-time and dynamic. Although the above methods can rapidly analyze the consequences of accidents, they did not integrate the real-time data acquired from accident scenes into accident models.

Some tools have been also developed or used in consequence assessment. Chrysoulakis et al. [5] developed a software tool that utilizes satellite images to automatically detect and monitor plumes caused by major industrial accidents. Qinqin et al. [6] developed a routine management system for environmental risk sources based on browser/server models and web-GIS technology. Oikonomou et al. [7] developed a self-calibrated wireless sensing system for monitoring the ambient industrial environment. These software packages focused on monitoring the hazards and accidents, but consequence assessment or analysis was not involved.

As mentioned, this paper proposes real-time data-based consequence simulation of pool fire triggered by MHI. On the basis of the established 3D model of pool fire according to the semi-empirical model proposed by the previous researchers, 3D visualization technology was brought into the simulation of the whole process of pool fire, including the dynamic flame and consequences that vary with the ambient parameters. The 3D visualization technology makes more spatial information utilized and obviously improves the objectivity and reality of pool fire simulation, which has effectively overcome the limitations of traditional two-dimensional technology. This simulation can provide some technical support for the government and enterprises on dealing with major accidents in order to reduce the accidents losses to the minimum.

\section{Three-dimensional Model of Pool Fire}

Pool fire mainly causes damage to the people in the direct vicinity and surrounding objects by the heat radiation of the flame. The extent of the damage to the surrounding objects depends on the 3D characteristics including geometric shapes and heat radiation flux. The 3D model of pool fire is established in the following sections which mainly describe the geometric shapes of flame varying with the real-time environmental parameters, and the heat radiation flux varying with the position information.

\subsection{Geometric Model of Pool Flame}

The geometric shape is designed to facilitate theoretical analysis and subsequent 3D visual consequence simulations, as shown in Fig. 1, combining with the three common flame geometries [8]-[10].

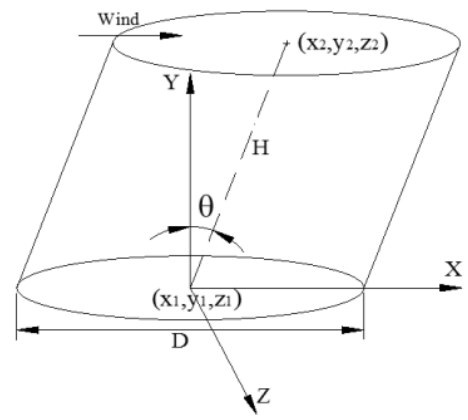

Fig. 1. Geometric shape of pool flame.

From the geometric shape in Fig. 1, the characteristic parameters include the pool diameter $D$, flame height $H$ and flame tilt angle $\theta$. Among them, the pool diameter $D(\mathrm{~m})$ can be calculated by

$$
D=2 \sqrt{W /\left(\pi H_{\min } \rho_{\text {liquid }}\right)}
$$


where $W(\mathrm{~kg})$ is the quantity of leaked liquid, $\rho_{\text {liquid }}\left(\mathrm{kg} / \mathrm{m}^{3}\right)$ the density of liquid and $H_{\min }(\mathrm{m})$ the minimum thickness of oil layer which is related to the characteristics of ground as shown in Table 1.

Table 1. Relationship between the Minimum Thickness of oil Layer and the Characteristics of Ground [11]

\begin{tabular}{cc}
\hline The characteristics of ground & $H_{\min }(\mathrm{m})$ \\
\hline Grassland & 0.020 \\
Rough ground & 0.025 \\
Flat ground & 0.010 \\
Concrete ground & 0.005 \\
The surface of calm water & 0.0018 \\
\hline
\end{tabular}

The flame height $H(\mathrm{~m})$ is defined by Thomas, 1963 and Thomas, 1965 [12] as follows.

$$
\begin{aligned}
& H=42 D\left(m_{f} /\left(\rho_{\text {Air }} \sqrt{g D}\right)\right)^{0.61} \quad \text { (without wind) } \\
& H=55 D\left(m_{f} /\left(\rho_{\text {Air }} \sqrt{g D}\right)\right)^{0.67}\left(U_{\text {wind }} /\left(g m_{f} D / \rho_{\text {Air }}\right)^{1 / 3}\right)^{-0.21} \\
& \text { (with wind) }
\end{aligned}
$$

where $\rho_{\text {Air }}\left(\mathrm{kg} / \mathrm{m}^{3}\right)$ is the density of air, $\mathrm{g}\left(\mathrm{m} / \mathrm{s}^{2}\right)$ the gravity acceleration, $U_{\text {wind }}(\mathrm{m} / \mathrm{s})$ the wind speed at height of 10 meters, if $U_{\text {wind }} \leq\left({ }^{g m_{f} D} / \rho_{\text {Air }}\right)^{1 / 3}, U_{\text {wind }} /\left(g m_{f} D / \rho_{\text {Air }}\right)^{1 / 3}=1$ [13]. $m_{f}\left(\mathrm{~kg} /\left(\mathrm{m}^{2} \cdot \mathrm{s}\right)\right)$ is the mass burning rate of the liquid, and it can be calculated as follows [14], [15].

$$
m_{f}=m_{\max }\left(1-e^{-k \beta D}\right)
$$

where $m_{\max }\left(\mathrm{kg} /\left(\mathrm{m}^{2} \cdot \mathrm{s}\right)\right)$ is the burning rate for an infinite diameter pool fire, $k\left(\mathrm{~m}^{-1}\right)$ absorption extinction coefficient of the flame and $\beta$ mean beam length corrector. The $m_{\max }$ and $k \beta$ values of several common oils are shown in Table 2.

Table 2. The Mmax and $\mathrm{k} \beta$ Values of Several Common Oils [16]

\begin{tabular}{ccccccc}
\hline Name & LNG & LPG & Benzene & Xylene & Gasoline & Kerosene \\
\hline$m_{\max }\left[\mathrm{kg} /\left(\mathrm{m}^{2} \cdot \mathrm{s}\right)\right]$ & 0.078 & 0.099 & 0.085 & 0.090 & 0.055 & 0.039 \\
$k \beta\left(\mathrm{m}^{-1}\right)$ & 1.1 & 1.4 & 2.7 & 1.4 & 2.1 & 3.5 \\
\hline
\end{tabular}

If the flammable liquid of single component is not listed in the Table 2, its rate of combustion can be calculated by

$$
m_{f}=0.001 H_{c} /\left(H_{\text {vap }}+C_{p}\left(T_{b}-T_{a}\right)\right) \quad\left(\mathrm{T}_{\mathrm{b}}>\mathrm{T}_{\mathrm{a}}\right)
$$




$$
m_{f}=0.001 H_{c} / H_{v a p}
$$

where $H_{c}(\mathrm{~J} / \mathrm{kg})$ is the combustion heat of the flammable material, $H_{\text {vap }}(\mathrm{J} / \mathrm{kg})$ the vaporization heat of the flammable material, $C_{p}(\mathrm{~J} /(\mathrm{kg} \cdot \mathrm{K}))$ the heat capacity, $T_{b}(\mathrm{~K})$ the liquid boiling temperature and $T_{a}(\mathrm{~K})$ the ambient temperature.

The flame tilt angle $\theta\left(^{\circ}\right)$ can be calculated by

$$
\theta=\arcsin \left(\left(\left(4 a^{2}+1\right)^{1 / 2}-1\right) /\left(1.332\left(U_{\text {wind }}^{2} / g D\right)^{0.333}\left(U_{\text {wind }} D / v_{\text {air }}\right)^{0.117}\right)\right)
$$

where $v_{\text {air }}\left(\mathrm{m}^{2} / \mathrm{s}\right)$ kinematic viscosity of air.

The geometric shape of flame, which varies with the environment parameters, directly affects the heat radiation flux of the flame surface. And the damage extent of pool fire can be obtained by the quantitative calculation of heat radiation flux.

\subsection{Quantitative Consequence Assessment of Heat Radiation Flux}

The heat radiation flux of the flame surface, $q_{0}\left(\mathrm{kw} / \mathrm{m}^{2}\right)$, is calculated as follows [17].

$$
q_{0}={ }^{0.25 \pi D^{2} H_{c} m_{f} f} /\left(0.25 \pi D^{2}+\pi D L\right)
$$

where $f$ is the thermal radiation coefficient, usually the empirical value of 0.15 .

The heat radiation flux received by the target, $q_{r}\left(\mathrm{kw} / \mathrm{m}^{2}\right)$, is related to the horizontal distance of the target to the center of the oil area. It can be calculated by

$$
q_{r}=q_{0}(1-0.058 \ln r) V
$$

where $r(\mathrm{~m})$ is the horizontal distance of the target to the center of the oil area and $V$ the view factor [8].

For people, the casualty probability $D_{p}$ can be calculated by [18].

$$
D_{p}=\int_{-\infty}^{P_{i}-5} e^{-u^{2} / 2} d u / \sqrt{2 \pi}
$$

where $P_{i}(i=1,2,3)$ represents a variable of different casualty extents, such as $P_{1}$ first-degree burns $\left(1^{\text {st }}\right.$ burn), $P_{2}$ second-degree burn ( $2^{\text {nd }}$ burn $)$ and $P_{3}$ death, which are described as follows. 


$$
\left\{\begin{array}{l}
P_{1}=-39.83+3.0186 \ln \left(t q^{4 / 3}\right) \\
P_{2}=-43.14+3.0188 \ln \left(t q^{4 / 3}\right) \\
P_{3}=-36.38+2.56 \ln \left(t q^{4 / 3}\right)
\end{array}\right.
$$

where $t(\mathrm{~s})$ is the time of exposure at the fire scene and $q\left(\mathrm{w} / \mathrm{m}^{2}\right)$ is the heat radiation flux received by a person in certain protective clothing equals the value of $400^{*} q_{r}$.

When people and objects are exposed at the fire scene, they have a certain limit of endurance. If the limit is exceeded, they will be hurt to a certain extent. Table 3 shows the critical value of heat radiation intensity corresponding to different extents of damage to human and objects.

Table 3. The Critical Value of Heat Radiation Intensity Corresponding to different Damage Extents [16]

\begin{tabular}{cccccc}
\hline Categories & \multicolumn{3}{c}{ Human } & \multicolumn{2}{c}{ Objects } \\
\hline Extents of damage & Mild burn & Severe burn & Death & Minor damage & Serious damage \\
Heat radiation intensity $\left(\mathrm{kw} / \mathrm{m}^{2}\right)$ & 4.0 & 12.5 & 25.0 & 12.5 & 25.0 \\
\hline
\end{tabular}

Based on Eq. (9) and the critical values of heat radiation intensity corresponding to different damage extents, the influence radius can be calculated by computer programming as shown in Fig. 2 . And the details of calculation are described as follows.

- Setting an initial value $r$ representing for the distance from the center of the oil area.

- Calculating the value of heat radiation flux by Eq. (9).

- $q_{c r}$ is the critical value corresponding to different extents of damage listed in Table 3. Comparing $q_{r}$ and $q_{c r}$ if $q_{r}$ is not equal to $q_{c r}, \mathrm{r}$ will be larger or smaller every step length $\Delta h$. Then $q_{r}$ is calculated again until it is equal to $q_{c r}$.

- Returning the result value $r$ as the influence radius corresponding to the extent of damage.

From the Section 2.1, the heat radiation flux is affected by the environmental parameters. So, the quantitative consequences in this section, such as the casualty probabilities and influence ranges, are also affected by the environment parameters. Besides, the casualty probabilities are related to the distance from the center of flame. The distance depends on the position information or coordinates of people at the fire scene. Therefore, the real-time data of the key parameters are greatly meaningful for the dynamical accident consequences.

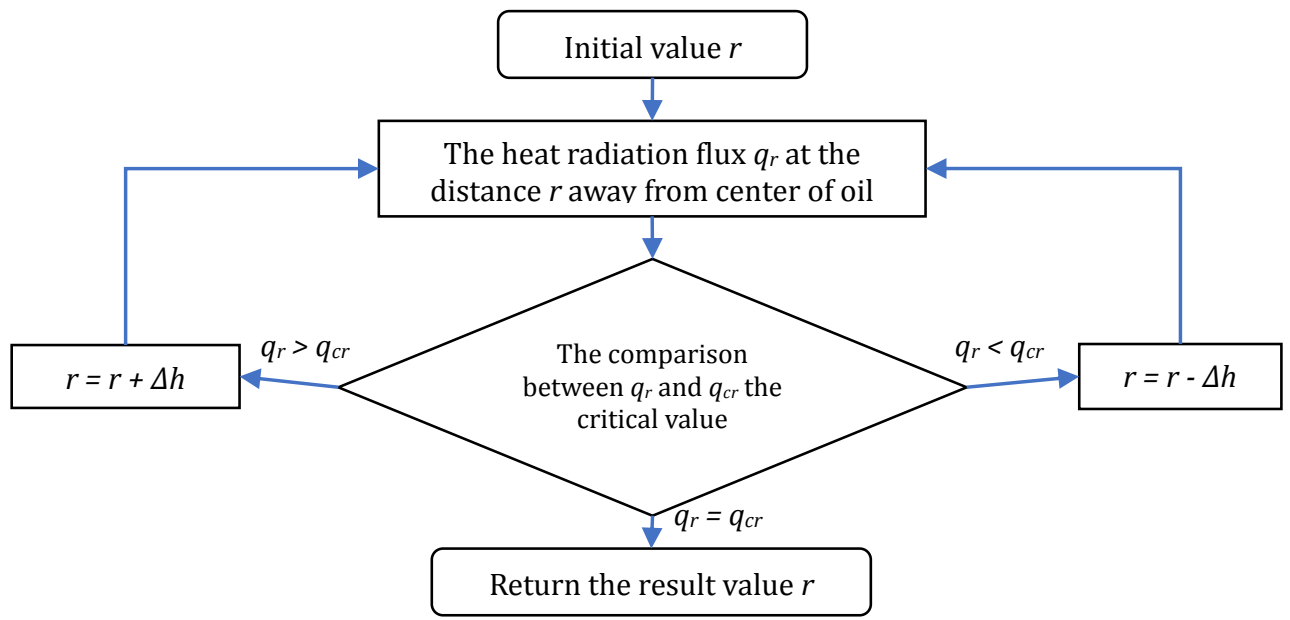

Fig. 2. The method of calculating the influence radius. 


\subsection{The Method of Acquiring Real-time Data}

The dynamical parameters in the above-established model, such as the wind speed, temperature and the position information of people, are concerned as the key parameters. And the real-time data of the parameters was acquired from a monitoring system published elsewhere [19], [20]. The monitoring system works with some sensors together to acquire the real-time data of environment parameters.

The parameters of the wind speed sensor are described as follows.

- The starting wind speed is less than $0.4 \mathrm{~m} / \mathrm{s}$.

- The measuring range is from 0 to $70 \mathrm{~m} / \mathrm{s}$.

- It is suitable for the ambient temperature from $-40{ }^{\circ} \mathrm{C}$ to $80^{\circ} \mathrm{C}$.

- It is suitable for the ambient humidity from 0 to $100 \% \mathrm{RH}$.

- The resolution ratio can reach $0.1 \mathrm{~m} / \mathrm{s}$.

A controlling device of LTM8662 is used to control different sensors in the lower computer, which communicates with the upper computer by the mode of RS485.

Besides, the position information or coordinates of people at the fire scene can be acquired from the following 3D simulation system of pool fire. The 3D simulation system designs a virtual human model. The coordinates of the virtual human can be displayed and acquired in real time.

After the real-time data is acquired, it will be transmitted and recalled in the 3D simulation system. On the basis of this, the 3D simulation system can dynamically analyze the real-time consequence of pool fire. And the following section shows the details of designing and developing the 3D simulation system of pool fire.

\section{Development of 3D Simulation System of Pool Fire}

\subsection{Overall Framework}

In line with the virtual reality (VR) idea, the 3D visualization simulation system is designed and implemented based on the 3D model of pool fire, integrating with the previously developed monitoring system. The main function modules of the 3D visualization simulation system of pool fire are shown in Fig. 3. The modules are 3D virtual scene management, consequence assessment and 3D dynamic simulation. 


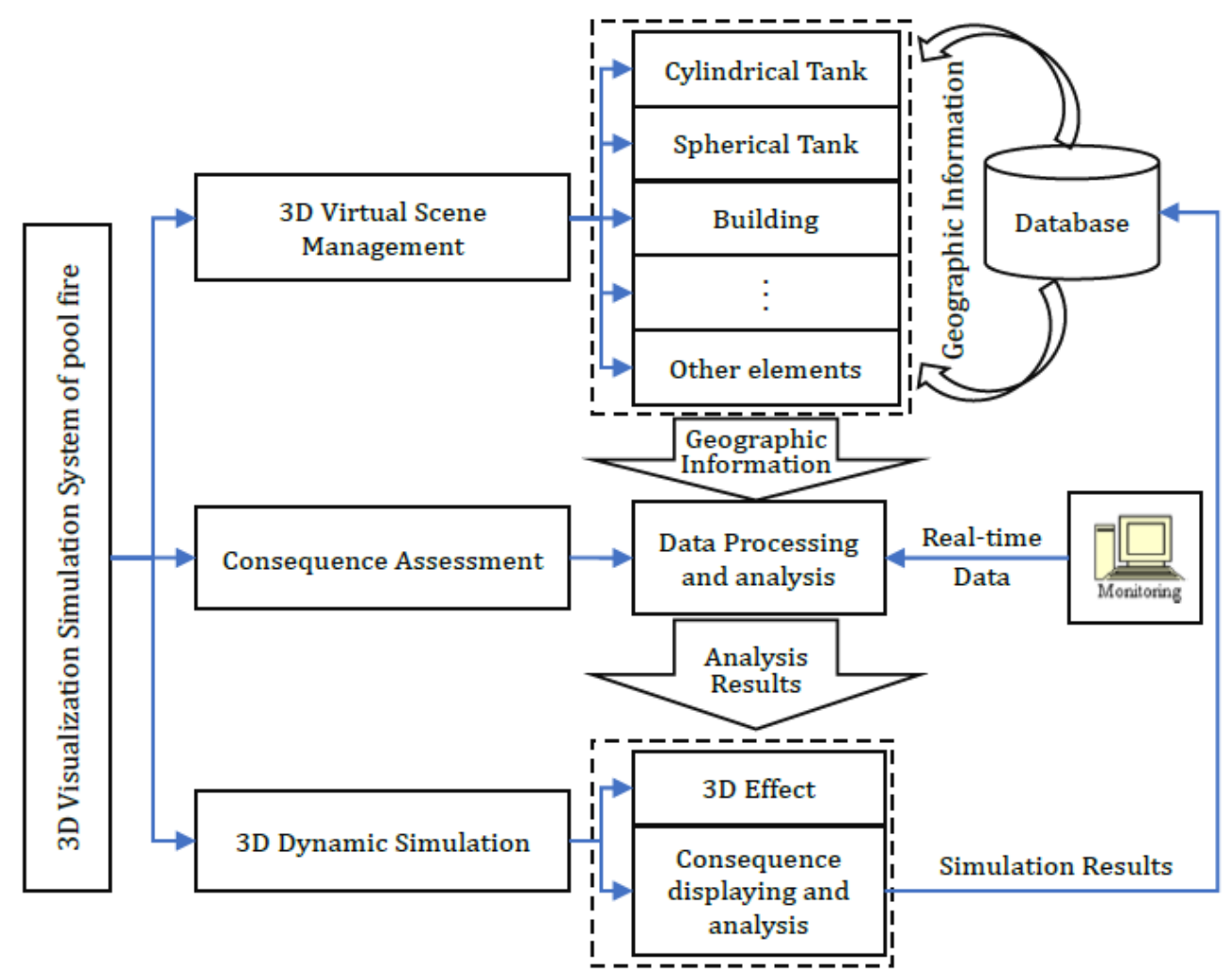

Fig. 3. Overall framework of 3D visualization simulation system of pool fire

The module of 3D virtual scene management reconstructs the accident scene composed of various hazard installations and other elements, providing the geographic information for other modules as a simulation container. The module of consequence assessment analyzes and processes the real-time data and geographic information to predict and evaluate the influence extent to the surrounding people and objects. The module of 3D dynamic simulation can display the consequence analysis results in the virtual accident scene. 


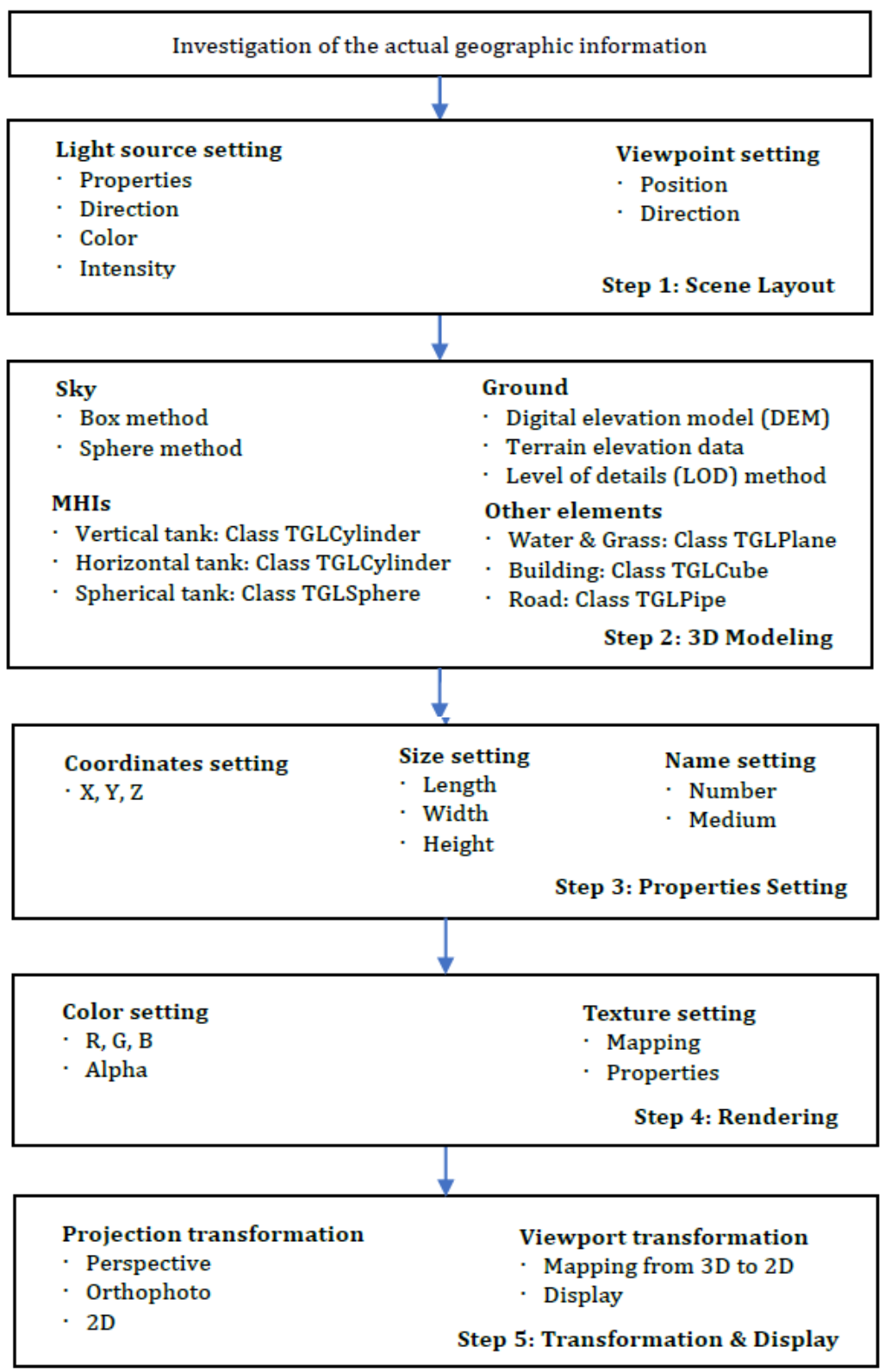

Fig. 4. The process of 3D entity modeling and rendering.

\subsection{Development Tools}

In accordance with the need for quick calculation and chart drawing, 3D visualization simulation system is developed by Delphi as the main programming tool to improve the efficiency of designing human-computer interaction ( $\mathrm{HCI}$ ) interfaces including acquiring real-time data and consequence analysis, and SQL Server as the database. The modules, such as 3D virtual scene management and 3D dynamic simulation, are developed by GLScene. GLScene is a component package of OpenGL and can decrease the complexity of OpenGL in large-scale 3D programming. Based on VR idea, GLScene is a perfect tool for 3D graphics programming in Delphi, considering the interactive modeling, expansibility, cross-platform and implementation level, etc. By utilizing GLScene, the following sections describe the details of implementing the modules shown in Fig. 3.

\subsection{Implementation of 3D Virtual Scene}

Reconstructing a 3D virtual scene is effectively the process of 3D entity modeling and rendering. The 3D 
scene usually includes geographic information, such as MHIs (vertical tank, horizontal tank and spherical tank, etc.) and the surrounding information (building, road, water, grass and other elements). The process of designing each geographic element is modularized in the 3D visualization simulation system. It is convenient for users to construct arbitrary 3D virtual scenes including main geographic information identical to the actual environment. Fig. 4 shows the development method and process of 3D entity modeling and rendering. The details of the method are described as follows.

- Step 1: laying out the virtual accident scene, such as setting the viewpoint and light source after investigation of the actual geographic information.

- Step 2: 3D entity modeling of the simulation container composed of sky, ground, MHIs, water, grasses, buildings, roads and so on.

- Step 3: Setting the properties of the entities, such as coordinates, size and name, etc.

- Step 4: Rendering of the 3D entity models by setting the properties of the color and texture.

- Step 5: Displaying the 3D models on the 2D screen by the projection transformation and viewport transformation.

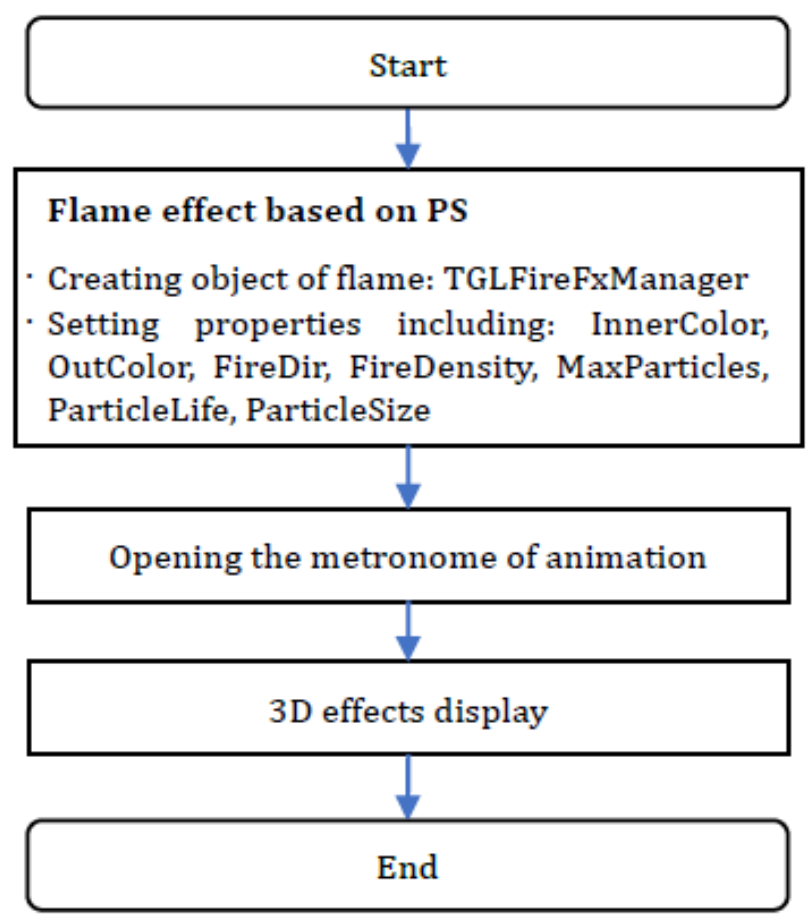

Fig. 5. The process of generating dynamic effects of pool flame.

\subsection{Implementation of 3D Dynamic Effect of flame}

The 3D dynamic effect of flame is implemented based on the particle system (PS) algorithm proposed by Reeves, 1983. The basic design idea of PS is describing irregular objects by utilizing many particles of simple shapes as the basic elements. It is possible to dynamically simulate complex natural phenomena, such as smoke, clouds, water, fire and so on [21]. GLScene encapsulates all the characteristics and advantages of PS. Fig. 5 shows the process of generating dynamic flame by PS in GLScene. The process is described as follows.

- Creating an object of TGLFireFxManager and setting the properties of flame particles.

- Opening the animation metronome that controls the dynamical effect of the flame.

- Displaying the 3D animation effect of pool fire. 


\subsection{D visualization of accident consequence}

The influence ranges can be calculated by the method shown in Fig. 2. To visually describe the influence extents in 3D visualization simulation system, Fig. 6 shows the method of drawing and displaying the influence ranges by GLScene. The details are as follows.

- Recalling the consequence analysis result $\mathrm{Ri}$ as the initial value of the influence range.

- Defining an object of TGLSphere and setting the properties to draw the influence range.

- Displaying the influence range in the virtual accident scene.

- Searching and outputting the list of objects involved within the influence range.

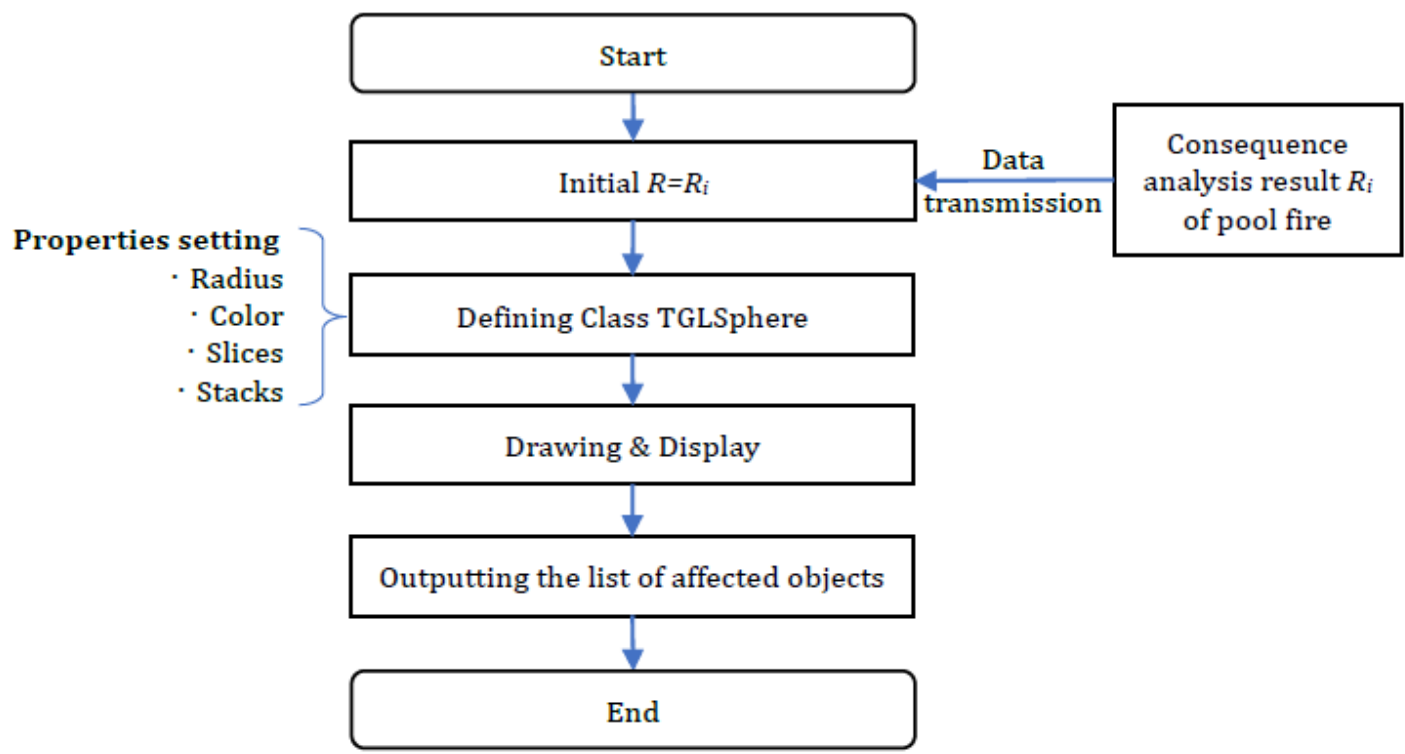

Fig. 6. The method of drawing influence ranges of pool fire.

\section{Case study and Analysis}

\subsection{Case Profiles}

This case will show how to simulate a pool fire triggered by a tank storing liquefied petroleum gas (LPG). This simulation test is used to validate whether the consequences can be predicted and evaluated on the basis of real-time data. There are five propane tanks with a volume of $3500 \mathrm{~m}^{3}$, five propane tanks with a volume of $3000 \mathrm{~m}^{3}$, three butane tanks with a volume of $10000 \mathrm{~m}^{3}$, one butane tank with a volume of 6500 $\mathrm{m}^{3}$ and two horizontal tanks with a volume of $100 \mathrm{~m}^{3}$ at the storage area of the enterprise. The natural conditions of the city in which the enterprise operates are as follows: annual mean wind speed equals 2.4 $\mathrm{m} / \mathrm{s}$, annual mean temperature equals $21.9^{\circ} \mathrm{C}$ and annual mean relative humidity equals $81 \%$.

\subsection{Simulation and Analysis}

(1) The virtual accident scene

To enhance the sensing cognition of pool fire, the virtual accident scene is reconstructed in Fig. 7 in accordance with the implementation process in Fig. 4. The implementation process is based on the 3D entity modeling and rendering of GLScene proposed in Section 3.2. By utilizing the tool of GLScene, the 3D visualization simulation system eventually simulates the geographic elements corresponding to the actual accident scene, such as major hazard installations (vertical tanks, cylindrical tanks and spherical tanks), buildings, road, green area, water area, street lamp and so on. Besides, the geographic elements are modularized in the implementation process. It not only improves the efficiency of reconstructing the virtual 
accident scene, but also enhances the ability that 3D visualization simulation system can simulate the arbitrary accident scene corresponding to the actual scene.

(2) The acquisition of real-time data

Based on the method proposed in Section 2.3, the monitoring system can acquire the real-time data from the accident scene by connecting different sensors. Fig. 8 shows the real-time temperature acquired from the actual scene. The temperature values can be acquired every 12 seconds. The latest temperature value is shown in Fig. 8, as well as the trend of temperature variation. The real-time data acquired from the monitoring system is transmitted to the consequence assessment module in the 3D simulation system of pool fire.

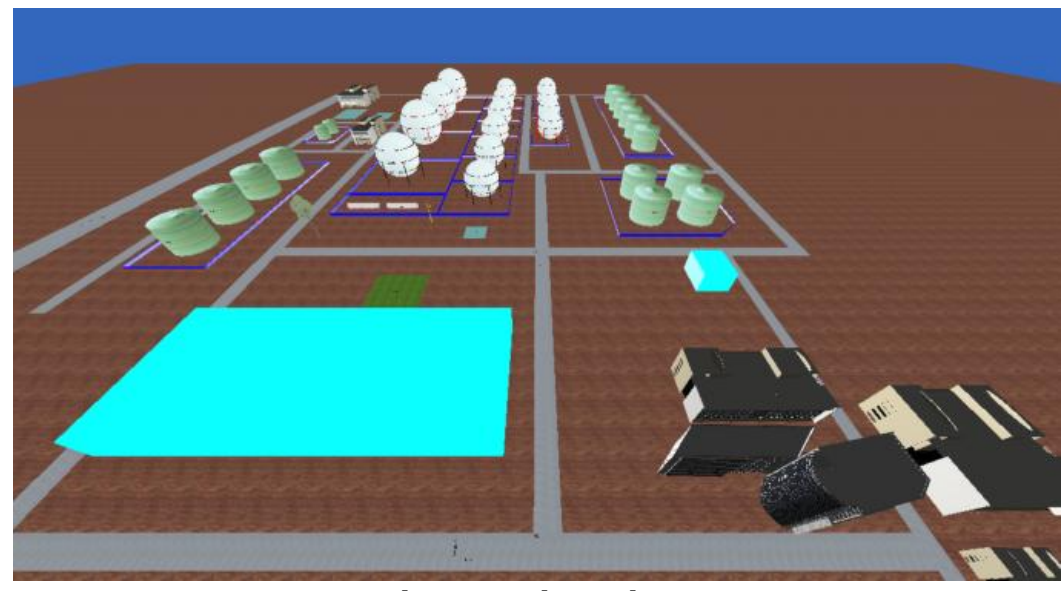

Fig. 7. The virtual accident scene.

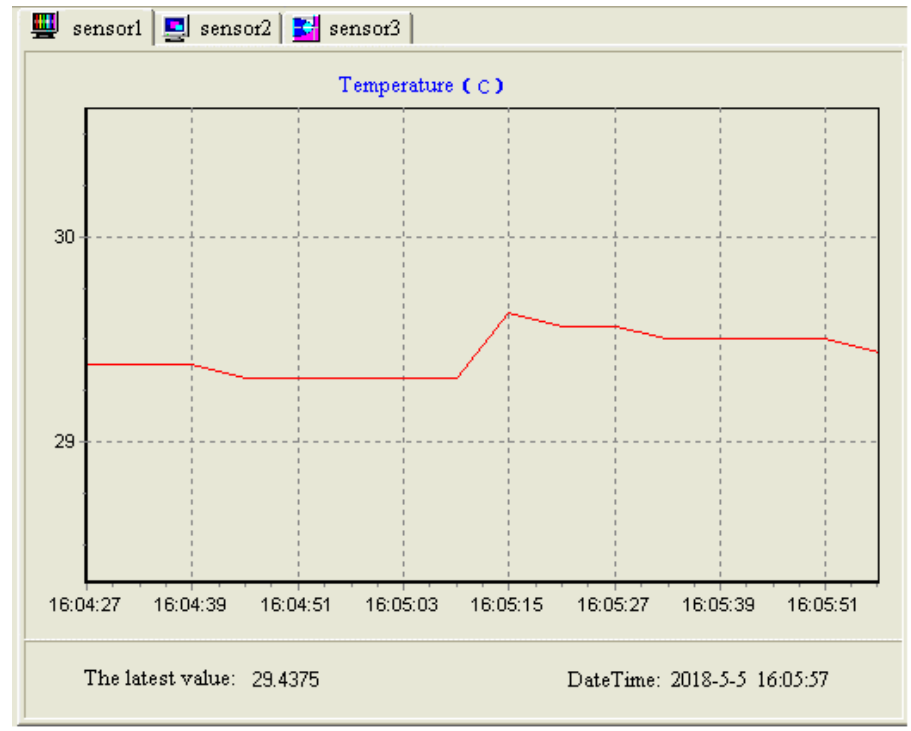

Fig. 8. The acquisition of real-time data.

(3) 3D dynamic effect of flame

The module of consequence assessment efficiently analyzes the 3D characteristics of pool fire based on the 3D model in Section 2.1. The geometric characteristics of the flame with different wind speeds are simulated in Fig. 9. According to Eq. (7) and the process of generating dynamic effect of flame in Fig. 5, Fig. 9(a) shows the flame shape without wind, and Fig. 9(b) shows the flame shape of tilt angle at a wind speed of $2 \mathrm{~m} / \mathrm{s}$. 


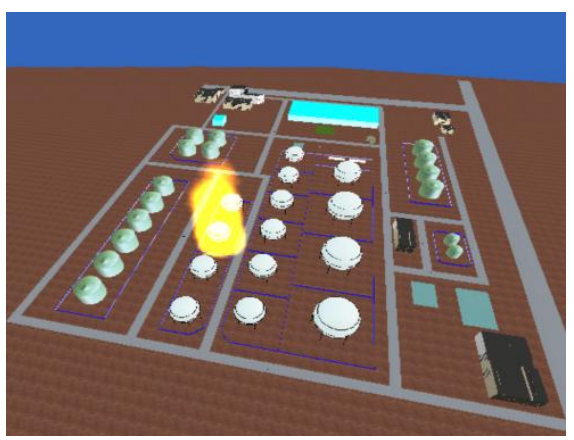

(a) Without wind

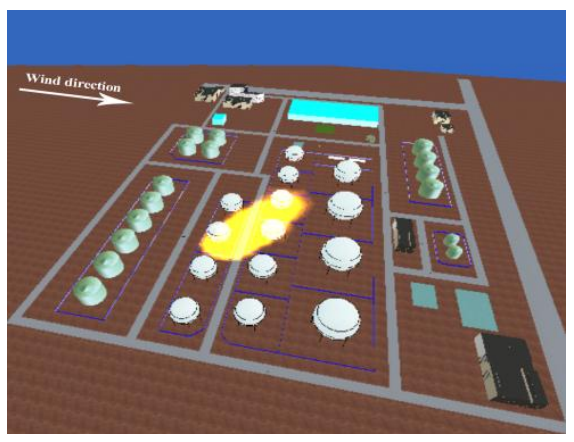

(b) Wind speed $=2 \mathrm{~m} / \mathrm{s}$

Fig. 9. The dynamical flame shapes with different wind speeds.

\section{(4) 3D simulation of accident consequence}

The hazard characteristics of heat radiation are analyzed and simulated as well based on the quantitative assessment method in Section 2.2 and the method in Fig. 6. Taking buildings at the accident scene as an example, Fig. 10 shows the influence range corresponding to the critical value $q_{c r}=25 \mathrm{kw} / \mathrm{m}^{2}$, which causes serious damage to the surrounding objects as shown in Table 3. The influence range is dynamically shown in the 3D scene with different wind speeds. Fig. 10(a) shows that the building, which is not involved in the influence range without wind, is in safety. However, the building is in danger when it is involved in the influence range with a wind speed of $2 \mathrm{~m} / \mathrm{s}$, as shown in Fig. 10(b). According to the 3D model of pool fire, the reason that the safe state of the building changes is that the flame shape varies with different wind speeds, so that the heat radiation flux changes as well.

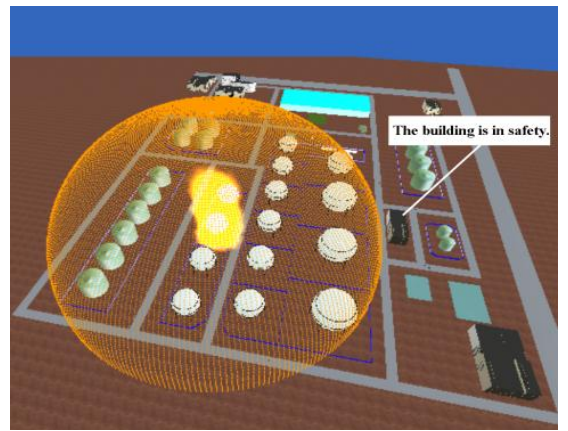

(a) Without wind

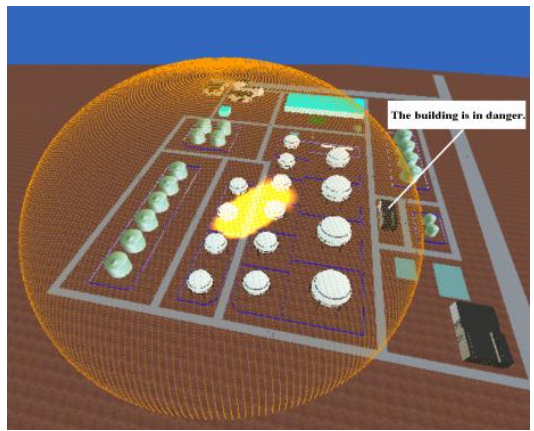

(b) With wind

Fig. 10. Influence range of heat radiation flux with different wind speeds.

For people, the system can also efficiently calculate the dynamic casualty probabilities at the accident scene, as shown in Fig. 11. Casualty probabilities depend on the distance $r$ according to Eq. (9)-Eq. (11). The distance is related to the position information of people in accident scene, which can be changed by moving the virtual people to simulate the behaviors of roaming in this 3D system. In this way, the casualty probabilities can be quickly calculated by Eq. (10), and the results are dynamically shown in the status bar of this 3D system as Fig. 11(a) and Fig. 11(b).

By roaming in the 3D scene and utilizing efficient calculations, some data on the relationships of casualty probabilities and distance is derived from this system and is shown in Fig. 12. According to the relationships in this simulation test, people at a distance of less than $17 \mathrm{~m}$ from the flame will be in danger of death. People at a distance of more than $30 \mathrm{~m}$ from flame will not be seriously burned, and the safety distance is $47 \mathrm{~m}$ from the center of flame. 


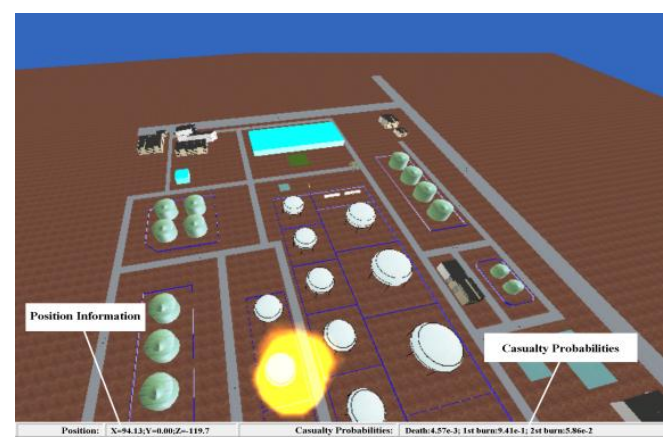

(a) Point 1

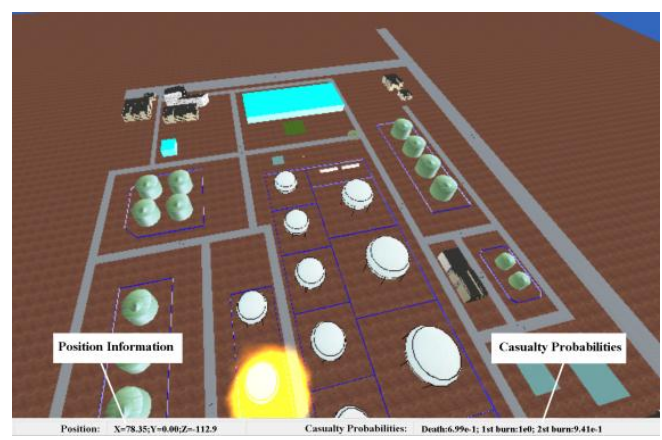

(b) Point 2

Fig. 11. Casualty probabilities at different positions.

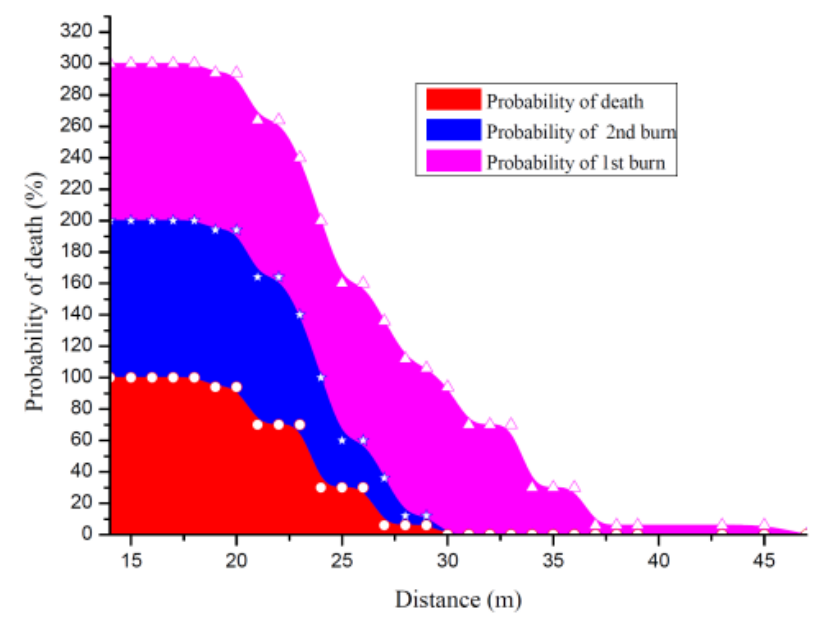

Fig. 12. Relationship of distance and casualty probabilities.

The relationships of heat radiation flux and distance with different wind speeds are drawn in Fig. 13 by efficient calculation of the 3D visualization simulation system. The results show that the target receives much more heat radiation at a wind speed of $2 \mathrm{~m} / \mathrm{s}$ than without wind speed. The heat radiation at a wind speed of $2 \mathrm{~m} / \mathrm{s}$ is less than the one at a wind speed of $4 \mathrm{~m} / \mathrm{s}$. From the results, the system developed in this paper can simulate the dynamic process of pool fire and evaluate the real-time consequences that vary with the ambient parameters.

\subsection{Discussion}

From the simulation results, the superiorities of 3D visualization simulation system of pool fire (3DVSS) include real time, high efficiency and immersive sense by comparing with the widely used commercial software packages, such as the 3D simulators based on computational fluid dynamics (CFD), and 2D simulators based on the classic calculation methods.

(1) Real time

By integrating with the developed monitoring system, 3DVSS has the functions of real-time monitoring and data transmission to ensure the real-time consequence assessment of pool fire that vary with dynamic parameters, so that the analysis results shown in Fig. 9 and Fig. 10 are more timeliness and objectivity. 3DVSS can not only simulate the accident consequences in a certain state like DNV software packages [17], but also simulate the dynamic consequences in any state. Thus, the real-time data-based consequence assessment is more meaningful than that without real-time data.

(2) High efficiency

From the calculation efficiency, it is well known that CFD will take much time to obtain the results 
through iterative operation based on a grid for the analysis of accidents. By comparison, 3DVSS has higher efficiency of analyzing the accident consequences than other 3D simulators based on CFD [22, 23]. Because 3DVSS obtains the analysis results through the basic mathematical operation based on the above established 3D model. Besides, compared with the 2D simulators, the 3DVSS can obtain the real-time analysis results without repeatedly setting state parameters, because the module of real-time monitoring can provide data sources acquired from accident scene. The high efficiency of real-time monitoring is helpful for accident prevention and prediction. And the high efficiency of consequence assessment is significant for taking emergency response measures to stop from the domino effect of accidents.

(3) Immersive sense

From Fig. 7, and Fig. 9-Fig. 11, 3DVSS that is developed based on VR idea can simulate the virtual scene corresponding to the actual circumstance, animation effects of accident occurrence and real-time consequences to systematically and vividly describe the hazard characteristics of pool fire, so that users have much more immersive sense. It will be helpful to enhance the sensing cognition of accidents. Thus, 3DVSS can not only be used in accident prevention and consequence assessment but also be used in safety education, training and fire drill.

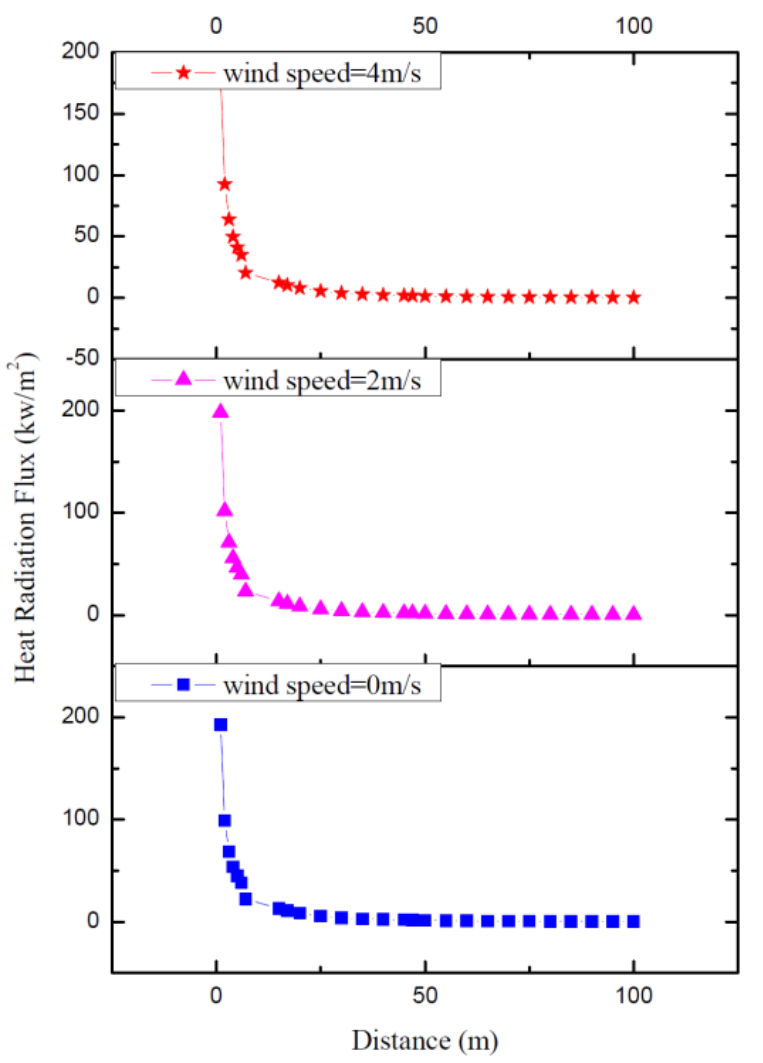

Fig. 13. Relationship of distance and heat radiation flux

\section{Conclusion}

The results of simulation tests show that heat radiation flux increases due to a higher intensity of combustion with a low wind speed, and heat radiation flux decreases due to the promotion of heat dissipation with a high wind speed. The simulation test proves that it is feasible to evaluate accident consequences based on real-time data, and it is reliable to describe the 3D characteristics of pool fire and the evolution trend of consequences varying with dynamic parameters. The method of consequence assessment for pool fire reflects not only efficient prediction and consequence evaluation at the current 
state, but also the evolution trend of consequences at the real-time and dynamic circumstances. Thus, it is greatly meaningful for the prevention of domino accidents.

Although 3DVSS is just applied in a petrochemical enterprise, it can be also applied in a chemical industry park. In order to enhance the applicability of the system, we will introduce artificial intelligence and internet of things to develop a distributed simulation system in the future, according to the distributed characteristics of MHI. In this case, the distributed simulation system can acquire the real-time data from regional or urban hazard installations. In addition, we will introduce virtual reality and augmented reality technologies to enhance the human-computer interaction of this system to further enhance immersive sense.

\section{Acknowledgment}

This work is supported by Jiangxi Provincial Department of Education in China (No. GJJ160083); Jiangxi Provincial Department of Science and technology in China (No. 20151BBG70058); National Natural Science Foundation of China (No. 21576102).

\section{References}

[1] Yu, Q., Jiang, J., \& Yu, H. (2012). Research on the emergency response system of major dangerous chemical accident on highway based on the GIS. Procedia Engineering, 45, 716-721.

[2] Adedigba, S. A., Khan, F., \& Yang, M. (2016). Dynamic safety analysis of process systems using nonlinear and non-sequential accident model. Chemical Engineering Research and Design, 111, 169-183.

[3] Khalil, Y. F. (2017). A probabilistic visual-flowcharting-based model for consequence assessment of fire and explosion events involving leaks of flammable gases. Journal of Loss Prevention in the Process Industries, 50, 190-204.

[4] Bubbico, R., \& Mazzarotta, B. (2018). Dynamic response of a tank containing liquefied gas under pressure exposed to a fire: A simplified model. Process Safety and Environmental Protection, 113, 242254.

[5] Chrysoulakis, N., Adaktylou, N., \& Cartalis, C. (2005). Detecting and monitoring plumes caused by major industrial accidents with JPLUME, a new software tool for low-resolution image analysis. Environmental Modelling and Software, 20(12), 1486-1494.

[6] Qinqin, C., Jia, Q., Yuan, Z., \& Huang, L. (2014). Environmental risk source management system for the petrochemical industry. Process Safety and Environmental Protection, 92(3), 251-260.

[7] Oikonomou, P. et al. (2016). A self-calibrated wireless sensing system for monitoring the ambient industrial environment. From lab to real-time application. Sensors and Actuators, B: Chemical, 237, 509520.

[8] TNO. (2005). Methods for the calculation of physical effects: Due to releases of hazardous materials (liquids and Gases): yellow book. Netherlands: Directorate-General for Social Affairs and Employment, Committee for the Prevention of Disasters.

[9] Witlox, H. (2005). POLF (Pool Fire) Theory Document.

[10] H. Witlox. (2006). PVAP Theory Document.

[11] Xu, Z., Wu, Z., \& He, J. (2007). Study on pool fire model applying to the safety assessment. Journal of Catastrophology, 22(4), 25-28.

[12] Raj, P. K. (2005). Large LNG fire thermal radiation - Modeling issues and hazard criteria revisited. Process Safety Progress, 24(3), 192-202.

[13] Ferrero, F., Muñoz, M., \& Arnaldos, J. (2007). Effects of thin-layer boilover on flame geometry and dynamics in large hydrocarbon pool fires. Fuel Processing Technology, 88(3), 227-235. 
[14] Roh, J. S., Yang, S. S., \& Ryou, H. S. (2007). Tunnel fires: Experiments on critical velocity and burning rate in pool fire during longitudinal ventilation. Journal of Fire Sciences, 25(2), 161-176.

[15] Hu, L. (2017). A review of physics and correlations of pool fire behaviour in wind and future challenges. Fire Safety Journal, 91, 41-55.

[16] Zhu, J., \& Chu, J. (2003). Calculation of characteristic parameters of pool fire and assessment of Its thermal radiation hazard. China Safety Science Journal, 13(6), 25-28.

[17] DNV. (2004). SAFETI v6.4 online help document. Norway.

[18] Shebeko, Y. N. et al. (1995). Some aspects of fire and explosion hazards of large LPG storage vessels. Journal of Loss Prevention in the Process Industries, 8(3), 163-168.

[19] Zhou, J., Chen, G., \& Chen, Q. (2008). Real-time data-based risk assessment for hazard installations Storing flammable gas. Process Safety Progress, 27(3), 205-211.

[20] Chen, W., Xu, S., \& Chen, G. (2009). Real-time monitoring system for major hazard installations based on HCI communication. China Safety Science Journal, 19(7), 119-123.

[21] Reeves, W. T. (1983). Particle systems---a technique for modeling a class of fuzzy objects. ACM SIGGRAPH Computer Graphics, 17(3), 359-375.

[22] Borg, A., Bjarne, P. H., \& Ove, N. (2014). The concept of validation of numerical models for consequence analysis. Reliability Engineering and System Safety, 125, 36-45.

[23] Masum, J., Muhammad, A. R., et al. (2015). LNG pool fire simulation for domino effect analysis. Reliability Engineering and System Safety, 143, 19-29.

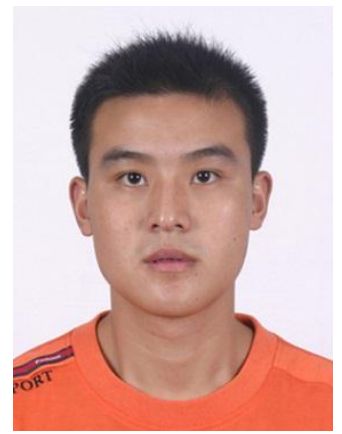

Wenjiang Chen received the B.Eng. degree in safety engineering from North China Institute of Science and Technology, China in 2007, and M.S. degree in safety technology and engineering from South China University of Technology, China in 2010, respectively. He is a lecturer of Nanchang University, China, where he is currently pursuing the Ph.D. degree. He is currently a visiting scholar at Florida Atlantic University, USA. His research and teaching interests include safety management information system, accident modeling, and consequence simulation based on computer technologies. 